\title{
Evaluation Of Incidental 18F-FDG Uptake Of Thyroid With Ultrasonography And Elastosonography
} \author{
Delibasi ${ }^{4}$
}

Esra Tutal1, Melia Karakose', Muyeser Sayki Arslan1, Mahmut Apaydın1, Ayşe Demirci $^{2}$, Taner Demirci ${ }^{1}$, Erman Cakal', Mustafa Sahin ${ }^{3}$, Mustafa Ozbek¹, Tuncay

1Diskapi Yildirim Beyazit Teaching and Research Hospital, Ankara, Turkey

2 Abdurrahman Yurtaslan Oncology Teaching and Research Hospital, Ankara, Turkey

${ }^{3}$ Ankara University, School of Medicine, Ankara, Turkey

${ }^{4}$ Hacettepe University (Kastamonu), School of Medicine, Ankara, Turkey

Thyroid incidentaloma detected by FDG-PET/CT (flourodeoxyglucose positron emition tomography/computed tomography) is important because of high rate of malignancy. Elastosonograpghy has proven valuable in discriminating malign and benign thyroid lesions. In this study, we aimed to evaluate PET incidentalomas by ultrasonography and elastosonography and to compare cytopathological results with imaging findings.

A total of 50 patients included into the study and maximum standardized uptake value (SUV max) values were recorded. All patients underwent laboratory evaluation (TSH, free T4, thyroglobulin antibody, thyroid peroxidase antibody and calcitonin) and underwent ultrasonography, elatosonography and fine needle aspiration cytology (FNAC). Cytopathological results were classified acoording to the Bethesda system. Elasticity of the suspicious nodule was scored as peviously described.

Of the 50 patients, 16 patients (32\%) were found diffuse uptake (group A) and 34 patients $(68 \%)$ were found focal uptake (group B). In group A, at least 1 antibody was found as positively $(100 \%)$ and thyroid nodule was detected in 9 patients (56\%). FNAC was revealed malignant cytology consistent with papillary carcinoma in 1 patient. In group $B$, thyroid nodule was detected in 31 patients. FNAC was revealed non-diagnostic in 4 patients, benign in 14 patients, atypia of undetermined significance in 2 patients, follicular neoplasm in 1 patient, suspicious for malignancy in 7 patients and malignant in 3 patients. Surgical resection was performed only in 4 patients and follicular carcinoma was revealed in 1 patient and papillary carcinoma was revealed in 3 patients. There was no statistically significant difference in the SUV max between benign and malignant nodules in both group $A$ and group $B$. There was a significant correlation between presence of malignancy and elasticity score $(r=0.756, p=0.000)$.

Increased elasticity score correlate with high likelihood of malignancy. Performing an elastosonography with ultrasonography may provide additional information to evaluate PET incidentalomas. 